\title{
CUILESS2016: a clinical corpus applying compositional normalization of text mentions
}

\author{
John D. Osborne' ${ }^{1}$ Matthew B. Neu¹, Maria I. Danila1', Thamar Solorio² and Steven J. Bethard ${ }^{3 *}$
}

\begin{abstract}
Background: Traditionally text mention normalization corpora have normalized concepts to single ontology identifiers ("pre-coordinated concepts"). Less frequently, normalization corpora have used concepts with multiple identifiers ("post-coordinated concepts") but the additional identifiers have been restricted to a defined set of relationships to the core concept. This approach limits the ability of the normalization process to express semantic meaning. We generated a freely available corpus using post-coordinated concepts without a defined set of relationships that we term "compositional concepts" to evaluate their use in clinical text.

Methods: We annotated 5397 disorder mentions from the ShARe corpus to SNOMED CT that were previously normalized as "CUI-less" in the "SemEval-2015 Task 14" shared task because they lacked a pre-coordinated mapping. Unlike the previous normalization method, we do not restrict concept mappings to a particular set of the Unified Medical Language System (UMLS) semantic types and allow normalization to occur to multiple UMLS Concept Unique Identifiers (CUIs). We computed annotator agreement and assessed semantic coverage with this method.

Results: We generated the largest clinical text normalization corpus to date with mappings to multiple identifiers and made it freely available. All but 8 of the 5397 disorder mentions were normalized using this methodology. Annotator agreement ranged from 52.4\% using the strictest metric (exact matching) to $78.2 \%$ using a hierarchical agreement that measures the overlap of shared ancestral nodes.
\end{abstract}

Conclusion: Our results provide evidence that compositional concepts can increase semantic coverage in clinical text. To our knowledge we provide the first freely available corpus of compositional concept annotation in clinical text.

Keywords: NLP, Information extraction, Concept normalization, Concept recognition, Fine grained named entity recognition

\section{Background}

Post-coordinated concepts are concepts represented by combining multiple concepts from an ontology, in contrast to pre-coordinated concepts, which are explicitly predefined and represented in an ontology by a single identifier. Post-coordinated concepts have been used by medical ontological systems such as GALEN [1] and SNOMED CT [2] to elucidate a broader range of concepts than is possible with pre-coordinated systems [3, 4] using descriptive logic. This methodology relies on a restricted set of pre-defined semantic relationships to avoid or min-

*Correspondence: bethard@email.arizona.edu

${ }^{3}$ School of Information, University of Arizona, 85721 Tucson, USA

Full list of author information is available at the end of the article imize semantic ambiguity. This is in contrast to Gene Ontology [5], which until the recent introduction of annotation extensions [6], assigned multiple annotations to a single protein without regard to the relationships between the assigned annotations. Not requiring formal semantic relationships for all multi-concept annotations may introduce some semantic ambiguity, but allows higher semantic coverage in situations where the source text describes a concept whose logical description cannot be captured by the set of pre-existing semantic relationships. Indeed, the ideal that an ontology of medicine can express "all and only what is medically sensible" has been termed "unobtainable" and focusing on "all" rather than "only" should take precedence [7]. 
In clinical interface systems utilizing SNOMED CT, complicated clinical concepts are typically created by clinicians who select from a set of inter-related atomic concepts with pre-defined relations. However the creation of a publicly available clinical text corpus with postcoordinated normalization training data has received less attention. This is likely due the difficulty and cost of creating and sharing such a corpus. Moreover, earlier work [8] comparing normalization between different SNOMED CT encoding groups that applied post-coordination to normalize text mentions in case report forms failed to find any statistically significant semantic agreement.

More recently, post-coordination has been applied in biomedical corpus construction with the creation of the NCBI Disease Corpus [9]. During corpus creation, Dŏgan first attempted to normalize disease mentions in PubMed abstracts to the MEDIC vocabulary using precoordinated concepts, which was successful for $91 \%$ of the disease mentions. For the remaining 9\% of disease mentions, they employed a minimally restricted form of post-coordination that we term "compositional" normalization that allowed the use of multiple concepts without regard to specific relations or "slots". They further categorized these "compositional concepts between "aggregate" or "composite" concepts that consisted of multiple self-contained pre-coordinated concepts in the text mention and "composed" concepts which collectively act to describe a single concept. The aggregate concepts in this context are simply concepts linked by logical operators (AND/OR) since no provision was made for logical operator usage in the annotation. Examples are shown in Table 1.

In the NCBI Disease corpus, only 76 such unique compositional concepts were normalized (52 aggregate and 24 composed) and annotator agreement for these postcoordinated concepts was not reported separately.

In contrast to the open-ended nature of Dŏgan's compositional concepts, Roberts [10] annotated postcoordinated concepts for only one predefined relation: anatomical location. Roberts' work includes both a corpus annotated on medical consumer language and software to normalize text mentions. However, the corpus contains only 500 post-coordinated concept instances.
SemEval-2015 Task 14 [11] annotated a corpus of clinical text with post-coordinated concepts, normalizing each disorder mention to a single SNOMED CT concept, and restricting further post-coordination to 8 predefined relations: body locations, which were normalized to UMLS anatomical concepts, and 7 other small-domain concept types. We refer to this corpus as "SEMEVAL2015". The SEMEVAL2015 section of Table 2 shows examples of each predefined relation. However, they report annotator agreement only for disorder mention normalization, not the overall normalization annotator agreement for that mention which would include associated postcoordinated concepts or slots. They were also unable to normalize $30 \%$ of the disorder mentions (such mentions are termed "CUI-less") because annotators were unable to find a single UMLS Concept Unique Identifier (CUI) for the concept. This suggests that there are limitations in the annotation process, the ontology being normalized to (SNOMED CT) or both, which prevent the full semantic capture of clinical text. This is known as the content completeness problem, first coined by Elkin $[12,13]$ but recognized earlier by Rogers and Rector [14].

In the current study we evaluate the extent to which compositional annotation, not restricted to a predefined set of relations, can attenuate the content completeness problem in clinical text. To address this problem, we generate the largest corpus to date for this compositional method. To our knowledge it is the first such compositional corpus in clinical text.

\section{Method}

\section{Corpus generation}

We generated a novel dataset "CUILESS2016" derived from the part of ShARe corpus used for the SemEval2015 Task 14 Shared Task [11], which we term, "SEMEVAL2015". Only a subset of SEMEVAL2015 was utilized, consisting of those disorder mentions that were not normalized to SNOMED CT, so called "CUI-less" disorders because they lack a Unified Medical Language System (UMLS) CUI corresponding to a SNOMED CT concept. Their distribution in the SEMEVAL2015 training and development datasets is shown in Table 3.

Table 1 Examples of pre-cordinated and post-coordinated concepts from the NCBI disease corpus

\begin{tabular}{|c|c|c|c|c|}
\hline Type / Subtype & & Identifiers & Text mention example & Concept name/s \\
\hline Pre-coordinated & & 1 & Bone dysplasia & Bone diseases, Developmental \\
\hline $\begin{array}{l}\text { Compositional } \\
\text { Aggregate (|) }\end{array}$ & / & 2 & Breast or ovarian cancer & Breast cancer|Ovarian cancer \\
\hline $\begin{array}{l}\text { Compositional } \\
\text { Composed }(+)\end{array}$ & / & 3 & Inherited neuromuscular disease & $\begin{array}{l}\text { Neuromuscular disease }+ \text { Genetic diseases }+ \\
\text { Inborn }\end{array}$ \\
\hline
\end{tabular}

Post-coordinated concepts of type ("aggregate" or "composed") have 2 or more identifiers 
Table 2 CUI-less examples from SEMEVAL2015 and CUILESS2016 annotation of ShARe corpus

\begin{tabular}{llll}
\hline & & Aggregate example & Composed example \\
\hline SEMEVAL2015 & Textmention & RRW & Surgical defect \\
Negation & Yes & Patient* & Patient* $^{*}$ \\
Subject & No* & Yes & Unmarked* \\
Uncertainty & Unmarked* & Unmarked* \\
Course & Unmarked* & False* \\
Severity & False* & False* \\
Conditional & False* & C1521748 (Entire mastoid) \\
Generic & C0225754 (Both lungs) & CUI-less \\
Body location CUI & CUl-less & C0543467 (Operative surgery) \\
Disorder CUI & C0034642 (Rhales) & C2004491 (Cicatrix)
\end{tabular}

An* indicates the default value for that slot in SEMEVAL2015. Our CUILESS2016 annotators added identifiers to describe the disorder when the Disorder CUI was marked "CUI-less" in SEMEVAL2015

We re-annotated only the CUI-less disorder CUI; CUIless body locations or other relations are not re-annotated, as shown in Table 2.

Since test data was not readily available, only disorder mentions from the development and training portion of SEMEVAL2015 were normalized. Approximately 30\% (5397) of disorder mentions fit this "CUI-less" description from a set of 298 training notes and a set of 133 development notes. The 298 training note set was itself derived from the notes used in the ShARe/CLEF eHealth 2013 Evaluation Lab Task 1 [15]. Statistics for the input SEMEVAL2015 corpus are provided in Table 4.

\section{Annotation method}

We used an open-ended compositional annotation methodology similar to that of Dŏgan [9] to normalize all 5397 "CUI-less" disorder mentions as described in the Annotation Guidelines (Additional file 1). Examples of our annotations are shown in the CUILESS2016

Table 3 SEMEVAL2015 CUI-less distribution by clinical document type

\begin{tabular}{llll}
\hline Data set & Document type & CUI-less count & $\begin{array}{l}\text { Average } \\
\text { CUl-less } \\
\text { by Note }\end{array}$ \\
\hline Development & Discharge summaries & 1929 & 13.9 \\
Training & Discharge summaries & 2796 & 20.6 \\
Training & Echocardiogram & 331 & 6.1 \\
Training & Electrocardiogram & 91 & 1.7 \\
Training & Radiology & 250 & 4.6 \\
\hline
\end{tabular}

Only discharge summaries were available for annotation in the development document set section of Table 2. Rules for annotation were similar to the ShARe/CLEF corpus [15] in that disorders were normalized to UMLS CUIs from SNOMED CT using the most specific CUI possible, ignoring negation and temporal modifiers, including acronyms, abbreviations and, to the fullest extent possible, mentions that are co-referent or anaphoric. There are some critical differences between the ShARe/CLEF annotation and our method that allow us to annotate these additional mentions. They are:

1 One or more identifiers were selected to annotate the text mention if (and only if) no appropriate single identifier (pre-coordinated term) is found.

2 All of SNOMED CT was available for mention normalization.

3 The annotators could use existing SEMEVAL2015 identifiers to create compositional concepts.

For example, if the mention "no bowel wall thickening" was annotated, and no CUI in SNOMED CT existed for "bowel wall thickening", but the SEMEVAL2015 annotations include a body location CUI for "bowel wall" and the disorder was flagged as negated, then the text mention was normalized using just the CUI for "Thickened

Table 4 SEMEVAL2015 and CUILESS2016 document statisticS

\begin{tabular}{llllll}
\hline \multirow{2}{*}{ Set } & Word count & \multicolumn{4}{l}{ Clinical note count } \\
\cline { 3 - 6 } & & Discharge & ECG & EKG & Radiology \\
\hline Train & $182 \mathrm{~K}$ & 136 & 54 & 54 & 54 \\
Development & $153 \mathrm{~K}$ & 133 & 0 & 0 & 0 \\
Total & $335 \mathrm{~K}$ & 269 & 54 & 54 & 54 \\
\hline
\end{tabular}


(fndg)", since the other two concepts needed for postcoordination are already present in the SEMEVAL2015 annotations.

Unlike the work of Dŏgan [9], we made no distinction as to whether the multiple CUIs used to annotate the span were aggregate or composed concepts. Thus, all of the CUIs in our mention were space separated and could represent either aggregation $(\mid)$ or concatenation $(+)$ per the operator nomenclature of Dŏgan [9].

\section{Calculation of annotator agreement}

Annotator agreement between the 2 annotators (MID and $\mathrm{MN}$ ) on the development data set was computed in 2 different ways.

1 Exact Agreement - Annotators used exactly the same set of CUIs to annotate the disorder text mention. We report only proportional agreement $p_{a}$ for this task by which we mean the fraction of text mentions on which the annotators agree. Thus, in Table 5 (in the Exact agreement row) we count only a single agreement for both Drug Allergy and Levofloxacin, not 2 agreements. Proportional agreement can be defined more formally as $p_{a}=m / n$ where $m$ is the number of mentions where both annotators agree and $n$ is the total number of mentions. This should approximate Cohen's $\kappa$ because agreement due to chance is expected to be extremely small. This is due to the UMLS representation of SNOMED CT having over 320K distinct CUIs and we allow an unbounded number of CUIs per mention.

2 Hierarchical Agreement - We compute hierarchical agreement between annotators using the set of annotated nodes and all their ancestors similar to the hierarchical precision and recall metric used by Verspoor [16]. It is calculated as:

$$
\frac{1}{n} \sum_{i=1}^{n}\left(\left\{\uparrow A_{i}\right\} \cap\left\{\uparrow B_{i}\right\}\right) /\left(\left\{\uparrow A_{i}\right\} \cup\left\{\uparrow B_{i}\right\}\right)
$$

where $\left\{\uparrow A_{i}\right\}$ indicates the set of annotated nodes and their ancestors from annotator A for mention i, $\{\uparrow B\}$ indicates the set of annotated nodes and their ancestors from annotator B for mention $i$ and $n$ is the total number of mentions annotated. In cases where an annotated CUI mapped to multiple SNOMED CT identifiers, SNOMED CT ancestors from all paths were used.

\section{Software and data}

Annotations were mapped using BRAT 1.3 software as shown in Fig. 1 [17]. Annotators SP, ES, MN and MID normalized the training data to the US Edition of SNOMED CT (2013_03_01) as represented in UMLS 2013AB. Development data was normalized to SNOMED CT (2016_03_01) in UMLS 2016AA by annotators MID and MN. Disorder CUIs found in the training data that were not present in SNOMED CT 2016_03_01 due to vocabulary changes or errors in the original annotation were normalized to SNOMED CT (2016_09_01) by MID and JDO.

\section{Results}

As shown in Table 6 we found the majority of disorder mentions had only a single identifier, which reflects the expanded range of available concepts and our guidance to use pre-coordinated concepts preferentially as outlined in our annotation guidelines. However Table 6 under-represents the true disorder multi-identifier count since disorder CUIs can be postcoordinated with SEMEVAL2015 annotations that represent disorder attributes. Thus "no bowel wall thickening" would be counted as "Single" in Table 6 since only the identifier for "Thickened (fndg)" was directly annotated; the anatomical CUI and negative polarity were already present in the linked SEMEVAL2015 attribute annotations.

Table 7 shows the overall distribution of disorderrelated identifiers both when attributes (non-disorder identifiers assigned in SEMEVAL2015) are either included or excluded from consideration. Thus in the

Table 5 CUILESS2016 annotator agreement type examples

\begin{tabular}{|c|c|c|c|c|}
\hline $\begin{array}{l}\text { Exact } \\
\text { mention } \\
\text { score }\end{array}$ & $\begin{array}{l}\text { Hierarchical men- } \\
\text { tion score }\end{array}$ & Text mention & Annotator 1 Concept/s & Annotator 2 Concept/s \\
\hline \multirow[t]{2}{*}{1.0} & 1.0 & Allergies Levofloxacin & Drug allergy & Drug allergy \\
\hline & & & Levofloxacin & Levofloxacin \\
\hline 0.0 & 0.52 & Posturing & $(\mathrm{O} / \mathrm{E})$ - posturing & Posturing behaviour \\
\hline \multirow[t]{2}{*}{0.0} & 0.64 & Rightward shift & Midline shift of brain & Midline shift of brain \\
\hline & & & To the right & \\
\hline 0.0 & 0.22 & Redness & Erythema & Redness \\
\hline
\end{tabular}

The computed hierarchical mention score was used instead of annotator judgment in determining an approximate level of agreement 


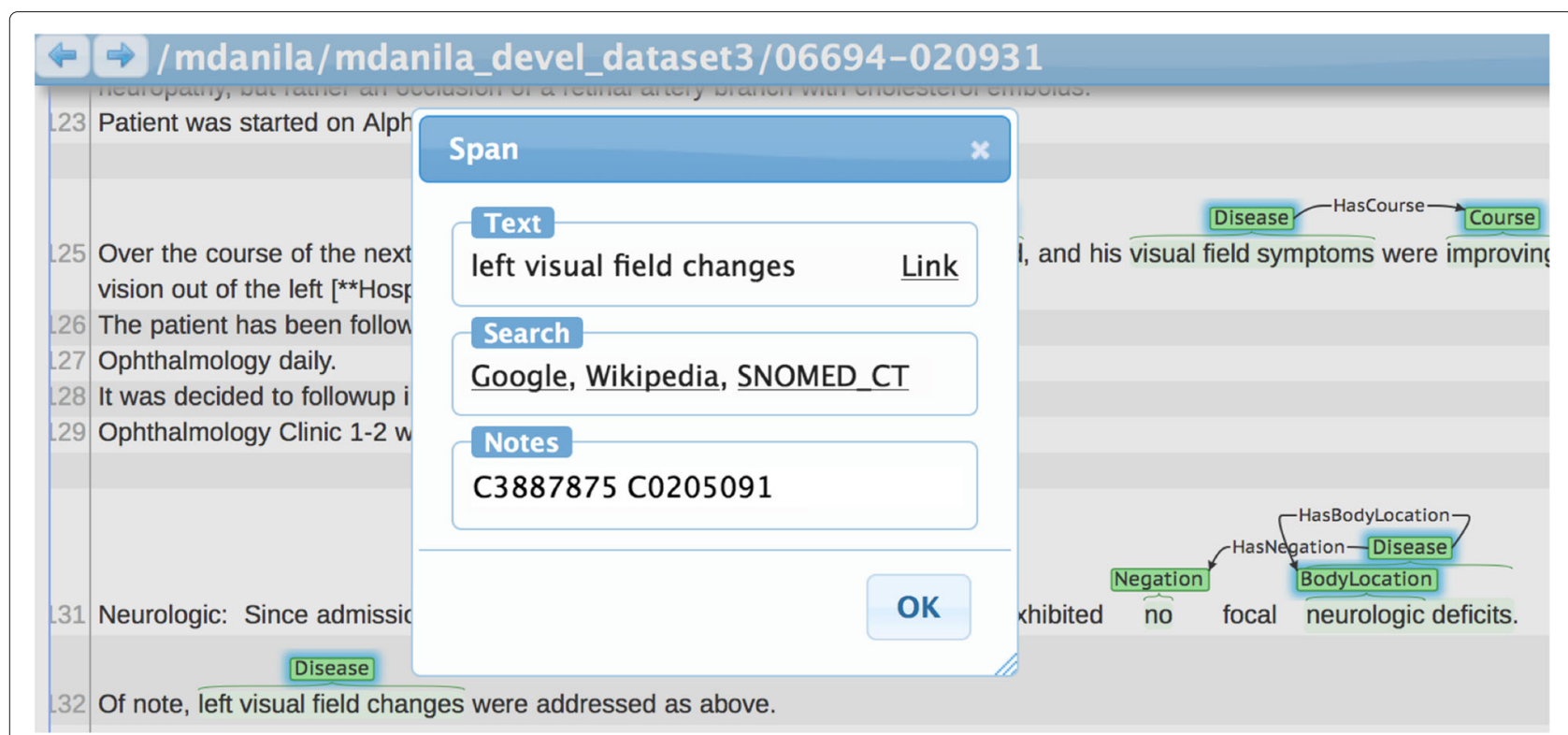

Fig. 1 Annotation Workflow. BRAT 1.3 [17] used to normalize concepts to UMLS CUIs from SNOMED CT

Disorder + Attributes column the text mention "no bowel wall thickening" was scored as having 3 identifiers, one for the disorder, one for the anatomical location and one for negation. Only when including these attributes are the majority of the concepts in CUILESS2016 post-coordinated.

Annotator agreement on the development set is shown in Table 8.

\section{Discussion}

We have normalized all but 8 of the 5397 original "CUIless" concepts in our corpus indicating that a compositional normalization methodology can alleviate the "content completeness problem" and increase semantic coverage in clinical text. All examples where our approach failed to normalize concepts are shown in Table 9. These examples fall into 3 general classes, those where the entity is not really a disease (named entity recognition failure), those where the text is ambiguous, and those where the annotators were unable to find a suitable composition in SNOMED CT. Only the last of these classes represents a concept that was truly not normalizable under our methodology. The 3 cases that fall into this class represent a tiny fraction $(0.06 \%)$ of the original 5397 mentions. Leveraging the existing SEMEVAL2015 annotation (which specified 8 different semantic modifiers of disorders) and allowing our annotators to normalize using a general semantic "association" (without specifying the exact relationship) allowed us to dramatically increase semantic coverage. Our corpus should be of interest to developers of clinical text normalization software interested in annotating a wider range of disorder annotations. We make our corpus freely available.

While our methodology is similar to that used by Dŏgan [9] for PubMed abstracts, we provide an order of magnitude more compositional normalization data. With the exception of some common abbreviations, the majority of compositional clinical concepts we created are composed

Table 6 Disorder multiple identifier distribution by data set

\begin{tabular}{|c|c|c|c|c|}
\hline Disorder CUI type & Development count & Development proportion & Training count & Training proportion \\
\hline CUI-less & 1 & 0.05 & 7 & 0.20 \\
\hline Single & 1687 & 87.46 & 2823 & 81.40 \\
\hline Double & 221 & 11.46 & 562 & 16.21 \\
\hline Triple & 18 & 0.93 & 73 & 2.11 \\
\hline Quadruple & 2 & 0.10 & 3 & 0.09 \\
\hline Total & 1929 & 100 & 3468 & 100 \\
\hline
\end{tabular}

Differences in disorder mention distribution between the development and training data set are likely due to note composition (see Table 3), a larger (4) set of annotators in the training data and a lack of a consensus process for the training data since each training document is annotated only by a single annotator 
Table 7 Overall disorder and attribute multiple identifier distribution

\begin{tabular}{llllll}
\hline \multirow{2}{*}{ Identifier type } & \multicolumn{2}{l}{ Disorder } & & \multicolumn{2}{c}{ Disorder + Attributes } \\
\cline { 2 - 3 } \cline { 5 - 6 } & Count & Proportion & & Count & Proportion \\
\hline CUI-less & 8 & $0.1 \%$ & & 3 & $0.06 \%$ \\
Single & 4502 & $83.54 \%$ & & 966 & $17.90 \%$ \\
Double & 783 & $14.53 \%$ & & 2505 & $46.41 \%$ \\
Triple & 91 & $1.7 \%$ & & 1608 & $29.79 \%$ \\
Quadruple & 5 & $0.1 \%$ & & 263 & $4.87 \%$ \\
Pentuple & 0 & $0.0 \%$ & & 50 & $0.93 \%$ \\
Hextuple & 0 & $0.0 \%$ & & 20 & $0.04 \%$ \\
Total & 5397 & $100 \%$ & & 5397 & $100 \%$
\end{tabular}

The Disorder column shows the count and proportion of disorders annotated with one or more concepts excluding attributes. The Disorder + Attributes column includes identifiers from attributes in the count to capture post-coordination with other identifiers

concepts, not aggregate concepts. This is in sharp contrast to Dŏgan [9] where the majority of mentions (114) from PubMed abstracts are aggregates of discrete concepts and only 34 mentions (24 unique) require logical description. Moreover, a substantial proportion (at least $16 \%)$ of the CUI-less clinical concepts required compositional normalization to specify the disorder mention. This is a higher proportion than is seen previously in PubMed abstracts [9] and consistent with the greater variability of clinical text.

\section{Exact annotator agreement}

There is a clear need for multi-identifier annotation in the clinical arena, where multiple identifiers are semantically critical for diseases such as cancer [18] and peripheral arterial disease [19]. However, evaluating the annotator agreement of post-coordinated concepts is difficult because of a lack of a common annotation standard. Previous studies reported proportionate agreement on exact matches $[8,15,20]$, but the definition of an "exact match" can vary.

For example Andrews [8], took research questions from case report forms and provided them to 3 different coding companies and instructed them to extract (normalize) core SNOMED CT concepts, using

Table 8 Development dataset annotator agreement

\begin{tabular}{lll}
\hline Agreement type & Agreement count & Proportionate agreement \\
\hline Exact & 1011 & 52.4 \\
Hierarchical & NA & 78.2 \\
Total mentions & 1929 & \\
\hline
\end{tabular}

There is no count for hierarchical agreement since each mention is assigned a value based on Eq. (1), whereas exact agreement assign every mention as a match (1.0) or not $(0.0)$
Table 9 Compositional CUI normalization error analysis

\begin{tabular}{ll}
\hline Mention & Error Class \\
\hline Allergies, Calcium & Named entity recognition failure \\
Atrial sensed & Named entity recognition failure \\
Left ventricular inflow pattern & Named entity recognition failure \\
RCIA & Ambiguous text \\
RC one Aneurysm & Ambiguous text \\
Echogenic kidney & No composition found \\
Making grammatical errors & No composition found \\
Tortous aorta & No composition found \\
\hline
\end{tabular}

All 8 mentions where annotators were unable to annotate the disease using the compositional approach

either pre-coordinated or post-coordinated expressions. Normalization was measured using proportionate agreement only at the "core concept" level, which ignored disagreements resulting from additional identifiers from modifiers. Even with this restriction, agreement between all 3 coding companies was calculated to be only 33\%, with $44 \%$ agreement between the two most similar annotation sets. Using Krippendorff's $\alpha$ as their statistic they concluded there was no significant semantic agreement in normalization. In contrast, our proportionate exact agreement (our worst performing metric) was $10 \%$ higher than their best inter-annotator agreement although we were more stringent in including disagreement to extend to non-core concepts. This may be due to their data set which was focused on rare diseases in case report forms (rather than clinical text), differences in the tool selection and/or annotator medical knowledge.

An alternative measure of annotator normalization agreement (accuracy) was used in the original annotation of this corpus [15] instead of Cohen's $\kappa$ and Krippendorf's $\alpha$. Annotator normalization agreement was calculated between annotators and was not separated from the underlying mention span boundary detection. A relaxed accuracy calculation where correctness was defined as any overlapping span where the disorder CUIs matched yielded an accuracy of 0.776, a "strict" agreement score based on exact span matching yielded a much higher agreement of 0.846 . However this high accuracy applies to single CUI disorder agreement. No annotator agreement was reported including disagreements with CUIs from the body location attribute or other included identifiers. While that reported "exact" agreement is higher than ours, we expected our agreement to be substantially lower since our annotation was for "CUI-less" disorders that they did not annotate. The original annotation deliberately excluded use of the UMLS semantic group finding for these disorders and reported that "this semantic group was found to be a noisy, catch-all category, and attempts 
to consistently annotate against it did not succeed in our preliminary studies."

\section{Non-exact annotator agreement}

Our exact agreement calculation cannot determine if a pre-coordinated concept and a post-coordinated concept are logically equivalent. Additionally, exact agreement cannot capture the difference between concepts with completely different meanings and hyponyms/hypernyms that have similar meanings. Our hierarchical agreement measure can account for this distinction. Hierarchical agreement penalizes distant errors and those at the higher levels of the hierarchy more severely than finer misclassifications, similar to hierarchical precision [16]. Unfortunately, the performance of hierarchical agreement is dependent on the structure of the ontology used. It is sensitive to the level of branching and assumes a consistent correlation between branch length and semantic distance. Thus even semantically similar concepts such as the posturing example seen in Table 5 may not score well, a consideration given the semantic duplication in SNOMED CT [21, 22]. We thus asked our annotators to consider the sets of concepts in each disagreement, and judge whether they were semantically equivalent, using their knowledge as medical professionals, rather than the exact structure of the ontology. The two annotators reached consensus easily on this task; there was only one case where they could not reach consensus, and for this, a neurologist was consulted to resolve the dispute. This process yielded a "semantic agreement" level of 71.6\%, 19\% increase over our exact agreement and is consistent with Casper [20] who reported $53 \%$ exact agreement and $75 \%$ semantic agreement.

\section{Compositional annotation rules}

One unresolved consideration with compositional annotations is which rules or conditions should govern annotation construction. In a previous study [8], the 3 coding companies mapping to SNOMED CT presumably (not specified in paper) used the extremely structured and elaborate SNOMED CT specific post-coordination specification to compose any post-coordinated diseases they annotated. However Pradhan [15] took a more general (but domain specific) approach specifying only 9 permissible disorder modifiers. All of these disorder specific domains (with the exception of body location) had a small (single digit) range of acceptable values. While core disorder concepts annotated in these publications should be comparable, associated concepts should be expected to be quite different. The more general annotation approach taken by Dŏgan [9] and this work allowed for any concept within the target ontology or ontologies. This allows for more flexibility at the expense of interpretation. For example, a body location CUI could refer to the site of disease finding, an affected organ, or a procedure site related to the illness. It is an open-ended question whether it is better to define the set of rules and allowable domains for post-coordination for each domain or to allow unrestricted composition. An enumerated set of possible relationships make closed world logic operations possible, but enumerating a complete and useful set of distinct semantic relationships that can be described in natural language text may not be feasible [7].

\section{Practical applications}

A practical application of our work is increasing semantic representation in clinical text. The approximately $70 \%$ coverage of named entities in SemEval-2015 Task 14 is too low for many practical purposes. Additionally, while SEMEVAL2015 corpus has the most exhaustive set of relations or slots for diseases to date, it still does not include important clinical relationships useful for practical applications of NLP. For example, metastasis, infection, surgical procedures or other SNOMED CT specified relations are relevant for practical clinical use. Additionally, by creating a corpus that includes clinical compositional annotation, this corpus opens the door to such annotation by machines that could potentially reduce the clinical coding burden.

\section{Limitations}

We have shown that annotating text from discharge summaries with compositional concepts from SNOMED $\mathrm{CT}$ is possible with high levels of annotator agreement. While this approach improves semantic coverage and is not bound to specific semantic relationship types, it does introduce a measure of semantic ambiguity since the relationship between the concepts is unclear. Thus, our annotations are more useful for information extraction than for logical reasoning, especially since we do not annotate logical operators (AND/OR) which would be useful in distinguishing aggregate from composite concepts. Future work should be able to make this distinction and also determine if our results are achievable for other medical text types (e.g., pathology reports) and other medical ontologies (e.g., the consumer health vocabulary).

We have shown high annotator agreement for annotating a single text mention with the identifiers of multiple ontological concepts, though we expect this agreement is lower than agreement on single identifier mentions. Unfortunately, we are unable to directly calculate single-identifier agreement because, under our annotation scheme, a mention which has been annotated with a single identifier may represent either (1) a true singleidentifier disease/disorder where the identifier completely captures the meaning, or (2) a disease/disorder where a single identifier captures only part of the meaning but 
the remaining meaning is captured by linked attributes (e.g., the body location already identified by the SemEval2015 Task 14 annotations).

\section{Conclusions}

In conclusion, we extended the SemEval-2015 Task 14 annotations of the ShARe disorder corpus to cover "CUIless" concepts and showed that the compositional annotation approach first used by Dŏgan [9] on PubMed text can function in clinical text to assign semantic identifiers to named entities and reduce the "content completeness problem" [12,13]. We believe our larger, freely available corpus is an important resource for the annotation of "CUI-less" concepts and that information extraction utilizing compositional normalization can lead to a more complete understanding of clinical text by complementing annotation approaches using predefined relations or slots such as the original ShareClef annotation. While annotation of complex clinical concepts using multiple identifiers has been routinely done by humans in a clinical or research setting, this corpus should aid the development of compositional normalization by machines to supplement manual coding practises.

\section{Additional file}

Additional file 1: Annotation Guidelines for Annotating CUI-less Concepts in BRAT. (PDF $1050 \mathrm{~kb}$ )

\section{Acknowledgements}

Shyam Patel and Efe Sahine helped annotate the training corpus. Discussions with Ken Barker were extremely helpful and his support in the creation of the annotation guidelines was much appreciated.

\section{Funding}

Research reported in this publication was supported by the National Institutes of Health. Support includes grant award number UL1TR001417 from the National Center for Advancing Translational Sciences and grant award number 1 R01GM1 14355 from the National Institute of General Medicine Science, "Extended Methods and Software Development for Health NLP". The content is solely the responsibility of the authors and does not necessarily represent the official views of the National Institutes of Health.

\section{Availability of data and materials}

To obtain a copy of the dataset it is required that the original ShARe corpus be downloaded first and a license agreement signed as described on the ShARe website (http://alt.qcri.org/semeval2015/task14/index.php?id=data-andtools). The CUI-less annotations may then be made downloaded from https:// physionet.org/works/CuilessClinical/. Annotation guidelines are included in the Additional Files section. An implementation of the hierarchical annotator agreement calculation for SNOMED CT can found in the HierarchicalAnnotatorAgreementClient class at https://github.com/ozborn/ jbratuimatools. All other intermediate data is available upon request.

\section{Authors' contributions}

JDO conceived the project idea, helped design the experiment, analyzed the data and wrote the first draft. SB and TS helped design the experiment, analyze the data, and edit the manuscript. MN and MID annotated the corpus, analyzed the data, and edited the manuscript. All authors read and approved the final manuscript.

\section{Ethics approval and consent to participate}

Not applicable, this study uses previously published de-identified human subject data that is classified as non-human subject data because of the lack of identifying information.

\section{Consent for publication}

Not applicable.

\section{Competing interests}

The authors declare that they have no competing interests.

\section{Publisher's Note}

Springer Nature remains neutral with regard to jurisdictional claims in published maps and institutional affiliations.

\section{Author details}

${ }^{1}$ University of Alabama at Birmingham, 7th Ave S, 1720 Birmingham, USA. ${ }^{2}$ Computer Science Department, University of Houston, Düsternbrooker Weg 20, 24105 Houston, USA. ${ }^{3}$ School of Information, University of Arizona, 85721 Tucson, USA.

Received: 10 April 2017 Accepted: 26 December 2017

Published online: 10 January 2018

\section{References}

1. Rector AL, Nowlan WA, Consortium G, et al. The galen project. Comput Methods Prog Biomed. 1994;45(1):75-8.

2. Spackman KA, Campbell KE, Côté RA. Snomed rt: a reference terminology for health care. In: Proceedings of the AMIA Annual Fall Symposium. Philadelphia: Hanley \& Belfus, Inc.; 1997. p. 640.

3. Elkin PL, Brown SH, Husser CS, Bauer BA, Wahner-Roedler D, Rosenbloom ST, Speroff T. Evaluation of the content coverage of snomed ct: ability of snomed clinical terms to represent clinical problem lists. In: Mayo Clinic Proceedings. Amsterdam: Elsevier; 2006. p. 741-8.

4. Hardiker NR, Rector AL. Modeling nursing terminology using the grail representation language. J Am Med Inform Assoc. 1998;5(1):120-8.

5. Ashburner M, Ball CA, Blake JA, Botstein D, Butler H, Cherry JM, Davis AP, Dolinski K, Dwight SS, Eppig JT, et al. Gene ontology: tool for the unification of biology. Nat Genet. 2000;25(1):25-9.

6. Huntley RP, Harris MA, Alam-Faruque Y, Blake JA, Carbon S, Dietze H, Dimmer EC, Foulger RE, Hill DP, Khodiyar VK, et al. A method for increasing expressivity of gene ontology annotations using a compositional approach. BMC Bioinformatics. 2014;15(1):155

7. Rector A, Rogers J. Ontological issues in using a description logic to represent medical concepts: Experience from galen. In: IMIA WG6 Workshop: Terminology and Natural Language in Medicine, Phoenix Arixona. 1999. http://www.opengalen.org/download/IMIAWG6-1999.pdf.

8. Andrews JE, Richesson RL, Krischer J. Variation of SNOMED CT coding of clinical research concepts among coding experts. J Am Med Inform Assoc. 2007;14(4):497-506.

9. Doğan Rl, Leaman R, Lu Z. NCBI disease corpus: a resource for disease name recognition and concept normalization. J Biomed Inform. 2014;47: $1-10$.

10. Roberts K, Rodriguez L, Shooshan SE, Demner-Fushman D. Automatic extraction and post-coordination of spatial relations in consumer language. In: AMIA Annual Symposium Proceedings. Bethesda: American Medical Informatics Association; 2015. p. 1083

11. Elhadad N, Pradhan S, Chapman W, Manandhar S, Savova G. SemEval-2015 Task 14: Analysis of clinical text. Proc Work Semant Eval. 2015;9:303-10.

12. Elkin $\mathrm{P}$, Bailey $\mathrm{K}$, Ogren $\mathrm{P}$, Bauer B, Chute C. A randomized double-blind controlled trial of automated term dissection. In: Proceedings of the AMIA Symposium. Philadel phia: Hanley \& Belfus, Inc.; 1999. p. 62-6.

13. Wong W, Liu W, Bennamoun M. Determining the unithood of word sequences using mutual information and independence measure. 2008;246-54. https://arxiv.org/abs/0810.0156.

14. Rogers J, Rector A. Terminological systems: bridging the generation gap. In: Proceedings of the AMIA Annual Fall Symposium. Philadelphia: Hanley \& Belfus, Inc.; 1997. p. 610

15. Pradhan S, Elhadad N, South BR, Martinez D, Christensen L, Vogel A Suominen H, Chapman WW, Savova G. Evaluating the state of the art in 
disorder recognition and normalization of the clinical narrative. J Am Med Inform Assoc. 2015;22(1):143-54.

16. Verspoor K, Cohn J, Mniszewski S, Joslyn C. A categorization approach to automated ontological function annotation. Protein Sci. 2006;15(6): 1544-9.

17. Stenetorp P, Pyysalo S, Topić G, Ohta T, Ananiadou S, Tsujii J. BRAT:a web-based tool for NLP-assisted text annotation. In: Proceedings of the Demonstrations at the 13th Conference of the European Chapter of the Association for Computational Linguistics. Stroudsburg: The Association for Computational Linguistics; 2012. p. 102-7.

18. Osborne JD, Wyatt M, Westfall AO, Willig J, Bethard S, Gordon G. Efficient identification of nationally mandated reportable cancer cases using natural language processing and machine learning. J Am Med Inform Assoc. 2016;23(6):1077-84.

19. Afzal N, Sohn S, Abram S, Liu H, Kullo IJ, Arruda-Olson AM. Identifying peripheral arterial disease cases using natural language processing of clinical notes. In: 2016 IEEE-EMBS International Conference on Biomedical and Health Informatics (BHI). New York: IEEE; 2016. p. 126-31.

20. Casper DS, Cimino JJ, Starren J. Reliability of SNOMED-CT coding by three physicians using two terminology browsers. In: AMIA Annual Symposium Proceedings. Amsterdam: Elsevier; 2006. p. 131-5.

21. Da Silveira M, Dos Reis J, Pruski C. Management of dynamic biomedical terminologies: current status and future challenges. Yearb Med Inform. 2015;10(1):125

22. Rogers J. Quality Assurance of Medical Ontologies. Methods Inf Med. 2006;45(3):267-74.

\section{Submit your next manuscript to BioMed Central and we will help you at every step:}

- We accept pre-submission inquiries

- Our selector tool helps you to find the most relevant journal

- We provide round the clock customer support

- Convenient online submission

- Thorough peer review

- Inclusion in PubMed and all major indexing services

- Maximum visibility for your research

Submit your manuscript at www.biomedcentral.com/submit 